\title{
Preparation of air-stable, low recombination velocity Si(111) surfaces through alkyl termination
}

\author{
William J. Royea, Agnes Juang, and Nathan S. Lewis ${ }^{\text {a) }}$ \\ Division of Chemistry and Chemical Engineering, 127-72, California Institute of Technology, Pasadena, \\ California 91125
}

(Received 8 May 2000; accepted for publication 27 July 2000)

\begin{abstract}
A two-step, chlorination/alkylation procedure has been used to convert the surface $\mathrm{Si}-\mathrm{H}$ bonds on $\mathrm{NH}_{4} \mathrm{~F}_{(\mathrm{aq})}$-etched (111)-oriented $\mathrm{Si}$ wafers into $\mathrm{Si}$-alkyl bonds of the form $\mathrm{Si}-\mathrm{C}_{n} \mathrm{H}_{2 n+1}(n \geqslant 1)$. The electrical properties of such functionalized surfaces were investigated under high-level and low-level injection conditions using a contactless rf apparatus. The charge carrier recombination velocities of the alkylated surfaces were $<25 \mathrm{~cm} \mathrm{~s}^{-1}$ under high-level and low-level injection conditions, implying residual surface trap densities of $<3 \times 10^{9} \mathrm{~cm}^{-2}$. Although the carrier recombination velocity of hydrogen-terminated $\mathrm{Si}(111)$ surfaces in contact with aqueous acids is $<20 \mathrm{~cm} \mathrm{~s}^{-1}$, this surface deteriorates within $30 \mathrm{~min}$ in an air ambient, yielding a high surface recombination velocity. In contrast, methylated $\mathrm{Si}(111)$ surfaces exhibit low surface recombination velocities in air for more than 4 weeks. Low surface recombination velocities were also observed for Si surfaces that had been modified with longer alkyl chains. (C) 2000 American Institute of Physics.
\end{abstract} [S0003-6951(00)01739-3]

It is well documented that the surface recombination velocity of $\mathrm{Si} / \mathrm{SiO}_{2}$ interfaces can be as low as $3 \mathrm{~cm} \mathrm{~s}^{-1}{ }^{1,2}$ Low surface recombination velocities are critical in a number of applications, including complementary metal-oxidesemiconductor devices ${ }^{3}$ and photovoltaics. ${ }^{4}$ Although ultrathin $\mathrm{Si}$ oxides are desirable for new generations of $\mathrm{Si}$ devices, control over the interfacial properties and thicknesses of ultrathin Si oxides is difficult. Passivation of Si surfaces will become even more important as device dimensions shrink and surface-to-volume ratios of electrical-device structures increase.

Hydrogen-terminated, (111)-oriented Si surfaces in contact with concentrated $\mathrm{H}_{2} \mathrm{SO}_{4(\mathrm{aq})}$, concentrated $\mathrm{HF}_{(\mathrm{aq})}$, and other acidic aqueous media exhibit very low surface recombination velocities. ${ }^{5}$ However, the surface recombination velocities $S$ of these surfaces degrade rapidly upon exposure to ambient air. Strategies to achieve potentially useful molecular-level control over the electrical properties of $\mathrm{Si}$ surfaces must therefore ensure that the resulting low $S$ persists in air for extended time periods. Crystalline Si has been functionalized previously at atmospheric pressure with organic layers through the use of alkyl Grignard and alkyl lithium reagents, ${ }^{6}$ alkenes, ${ }^{7-9}$ and phenyldiazonium salts, ${ }^{10}$ while the reaction chemistry of porous Si has been developed yet further to include use of alkynes ${ }^{11,12}$ and organohalides. ${ }^{13}$ The electrochemical properties of some of these surfaces have been reported, ${ }^{14,15}$ but to date there appears to be no information on the electrical properties of such systems. We report herein that crystalline $\mathrm{Si}$ functionalized through a twostep, wet-chemistry-based chlorination/alkylation procedure ${ }^{6}$ has an extremely low surface recombination velocity. Furthermore, the electrical passivation persists for extended time periods even for surfaces in contact with ambient air.

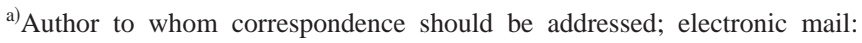
nslewis@its.caltech.edu
}

Long bulk lifetime, (111)-oriented float-zone Si wafers were used to investigate the effects of various surface treatments on the electrical properties of the $\mathrm{Si}$ surface. Samples $\approx 1.0 \mathrm{~cm}^{2}$ in area were cut from $3800 \Omega \mathrm{cm}$ resistivity, $\approx 190 \mu \mathrm{m}$ thick Si wafers that were polished on both sides. A micrometer was used to determine the thickness of each wafer to within a precision of $\approx 1 \%$. The samples were then etched in $40 \% \quad \mathrm{NH}_{4} \mathrm{~F}_{(\mathrm{aq})}$ to yield $\mathrm{H}$-terminated $\mathrm{Si}$ surfaces. ${ }^{16}$ Following the etch, no signals for elements other than silicon and (adventitious) carbonaceous material were observed in the wide scan of an X-ray photoelectron (XP) spectrum, and no oxide was detectable in the $\mathrm{Si} 2 p$ region. The H-terminated Si samples were then chlorinated using $\mathrm{PCl}_{5}$ in chlorobenzene, and were subsequently treated with either $\mathrm{CH}_{3} \mathrm{MgBr}$ (to obtain methylated surfaces) or with $\mathrm{C}_{8} \mathrm{H}_{17} \mathrm{MgBr}$ (to obtain octylated surfaces), as described previously. ${ }^{6}$ The XP spectra of the chemically modified surfaces indicated the absence of oxidized $\mathrm{Si}$, as well as increases in carbon levels for the longer chain alkane, consistent with prior XP measurements of such systems. ${ }^{6}$

Surface recombination velocity measurements were performed using a contactless rf conductivity apparatus. ${ }^{5,17-20}$ In this system, the output from a signal generator operating at $450 \mathrm{MHz}$ was connected to a power splitter. One output from the power splitter was connected through an amplifier and a phase shifter to the local oscillator input of a doublebalanced frequency mixer, and the other output from the power splitter was connected through an amplifier to the coupled port of a directional coupler. An inductor-capacitor $(L C)$ circuit consisting of a variable coupling capacitor, a variable matching capacitor, and a three-turn coil, placed in close proximity to the sample, was connected to the input port of the directional coupler. The output port of the directional coupler was then connected to the reference oscillator port of the double-balanced frequency mixer, and the output of the double-balanced frequency mixer was connected to a 


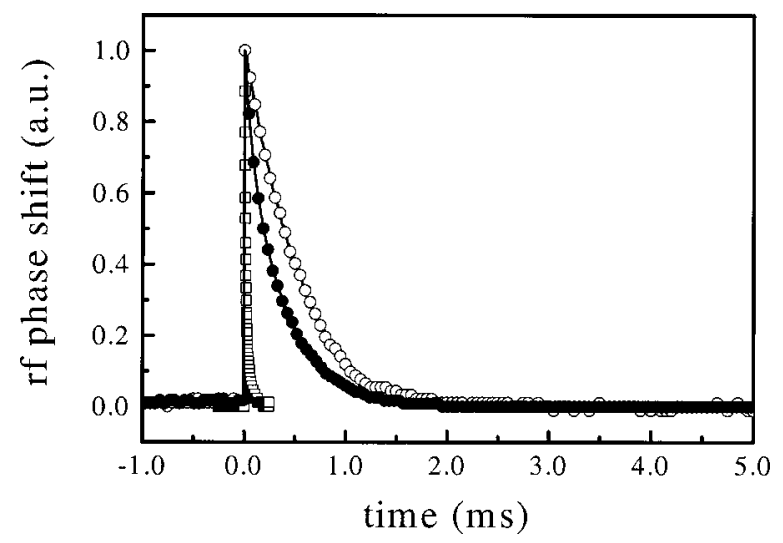

FIG. 1. Time-resolved photoconductivity decay of hydrogen-terminated (111)-oriented $n$-type $\mathrm{Si}$ in contact with concentrated sulfuric acid (unfilled circles) and after exposure to air for $30 \mathrm{~min}$ (unfilled squares). A singleexponential fit to these decays (not shown) yielded a time constant of $491 \mu \mathrm{s}$ for the $\mathrm{H}_{2} \mathrm{SO}_{4}$-immersed sample and $14 \mu \mathrm{s}$ for the air-exposed sample. Measurements were made under low-level injection conditions $\left(4 \times 10^{13}\right.$ injected carriers $\mathrm{cm}^{-2}$ into a $190 \mu \mathrm{m}$ thick $\mathrm{Si}$ sample). Also shown is a time-resolved photoconductivity decay of methylated (111)-oriented $n$-Si in air under high-level injection conditions $\left(2 \times 10^{16}\right.$ injected carriers $\mathrm{cm}^{-2}$ in a $190 \mu \mathrm{m}$ thick substrate) after $504 \mathrm{~h}$ in an air ambient (filled circles). A single-exponential fit to this decay (not shown) yielded a time constant of $342 \mu \mathrm{s}$.

digital oscilloscope for measurement of the photoconductivity decay signals. Prior to each measurement, the $L C$ circuit was tuned to the resonant frequency of the sample by adjusting the variable capacitors and monitoring the amplitude of the reflected rf signal on a separate high frequency digital oscilloscope.

Samples were illuminated using $10 \mathrm{~ns}$ pulses from a Nd:yttrium-aluminium-garnet laser (1064 nm) operating at a repetition rate of $10 \mathrm{~Hz}$. The power density of the beam was attenuated using a beam splitter and neutral density filters, and the beam was expanded to approximately $3 \mathrm{~cm}^{2}$ using a Galilean beam expander. A holographic diffuser placed directly above the sample was used to produce a spatially uniform beam profile on the Si. Using the neutral density filters, the power density of the expanded incident beam was adjusted to either $2 \times 10^{-3} \mathrm{~mJ}$ pulse ${ }^{-1}$ for high-level injection conditions or $3 \times 10^{-6} \mathrm{~mJ}$ pulse ${ }^{-1}$ for low-level injection conditions. The sample was placed into a sealed glass vessel so that the sample could be placed in contact with air, $\mathrm{N}_{2}(g)$, or various liquid solutions during measurement of the charge carrier lifetime. Time constants were obtained by fitting the average of 128 decays to a single exponential and averaging over a minimum of three samples for each type of surface at each measurement interval.

Figure 1 shows a representative rf conductivity decay signal of (111)-oriented, H-terminated $\mathrm{Si}$ in contact with concentrated $\mathrm{H}_{2} \mathrm{SO}_{4(\mathrm{aq})}$ under low-level injection. The decays were very slow, and could be fit well by a single exponential. The mean decay lifetime of such samples was $490 \pm 50 \mu \mathrm{s}$ under low-level injection and $800 \pm 100 \mu$ s under high-level injection. These values are consistent with the manufacturer's specification that the minority carrier lifetime in these wafers is $>200 \mu \mathrm{s}$. The observed lifetime $\tau$ can be related to the bulk lifetime $\tau_{b}$ and surface recombination velocity $S$ through the following: $:^{5,18,21}$ tylated Si surface exhibited lifetimes $(310 \pm 90 \mu \mathrm{s}$ under
Downloaded 04 Apr 2006 to 131.215.240.9. Redistribution subject to AlP license or copyright, see http://apl.aip.org/apl/copyright.jsp

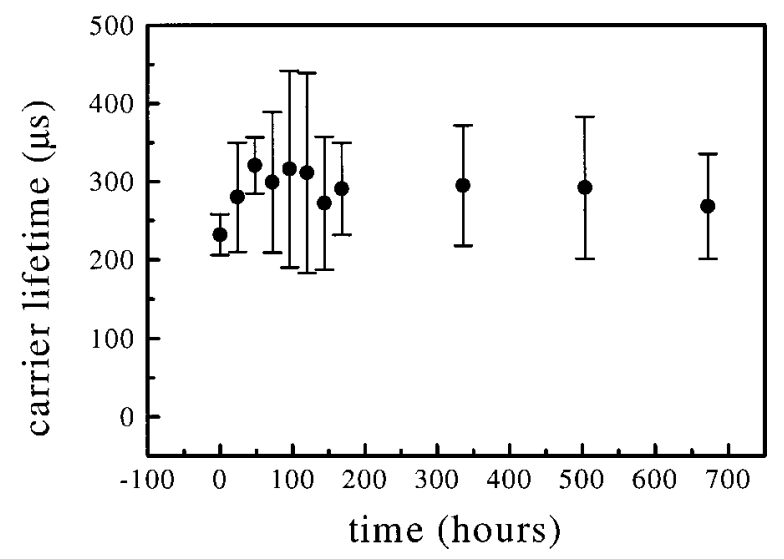

FIG. 2. Time dependence of the mean carrier lifetime for methylated Si in contact with air. Between measurements, the samples were stored in the dark at room temperature. All measurements were performed with a light pulse sufficient to provide high-level injection conditions during the initial carrier decay dynamics. The error bars represent $1 \mathrm{sd}$ values of the mean lifetimes in various measurements.

$$
\frac{1}{\tau}=\frac{1}{\tau_{b}}+\frac{2 S}{d},
$$

where $d$ is the sample thickness. Assuming that the experimentally observed lifetime is dominated by surface recombination implies that $S=19 \pm 2 \mathrm{~cm} \mathrm{~s}^{-1}$ under low-level injection and $12 \pm 2 \mathrm{~cm} \mathrm{~s}^{-1}$ under high-level injection. Of course these values are an upper bound on the true surface recombination velocity because they assume that the observed lifetime is dominated only by surface recombination, with no contribution from charge carrier recombination in the bulk of the sample.

Subsequent immersion of these samples in $48 \% \mathrm{HF}_{(\mathrm{aq})}$ produced somewhat shorter lifetimes of $180 \pm 60 \mu \mathrm{s}$ under low-level injection and $460 \pm 90 \mu$ s under high-level injection. These observations are in accord with the prior observations of Yablonovitch et al. that $S$ on H-terminated, (111)oriented $\mathrm{Si}$ surfaces is a function of the acidity of the electrolyte. ${ }^{5}$ Exposure to $36 \mathrm{M} \mathrm{H}_{2} \mathrm{SO}_{4(\mathrm{aq})}$ solutions reversed the decline in surface lifetime. However, exposure of this surface to air produced a rapid decay in the carrier lifetime, with lifetimes as short as $10 \mu$ s observed within $2 \mathrm{~h}$ of exposure to an atmospheric ambient (Fig. 1). The small $\tau$ value was persistent in air, and a long lifetime could only be recovered by etching the Si surface and/or by reimmersion of the crystal into a highly acidic aqueous electrolyte. This behavior was observed under both low-level and high-level injection conditions.

Figure 1 also depicts the behavior of the methylated $\mathrm{Si}$ surface in air. The observed lifetime was $260 \pm 50 \mu$ s under low-level injection and $290 \pm 80 \mu$ s under high-level injection, implying surface recombination velocities of $17 \pm 7$ and $21 \pm 9 \mathrm{~cm} \mathrm{~s}^{-1}$ for low-level and high-level injection, respectively. In addition, as shown in Fig. 2, these lifetimes were stable in ambient air for extended time periods, with no degradation in lifetime observed for the methylated $\mathrm{Si}$ surface after at least 30 days in an air ambient.

Similar experiments were performed with (111)-oriented $\mathrm{Si}$ surfaces that had been treated with $\mathrm{H}_{17} \mathrm{C}_{8} \mathrm{MgBr}$ instead of with $\mathrm{CH}_{3} \mathrm{MgBr}$. After $48 \mathrm{~h}$ of exposure to room air, an oc- 
low-level injection and $300 \pm 100 \mu$ s under high-level injection), and hence $S$ values, that were essentially identical to those of the methylated surfaces. A monolayer comprised of long alkyl chains could potentially act as an improved hydrophobic barrier towards oxidation, thereby enhancing the surface stability relative to methylated Si surfaces. However, due to methylene-methylene repulsions, alkyl chains longer than one carbon cannot be used to cover every topmost $\mathrm{Si}$ atom on the (111)-oriented Si surface. ${ }^{22}$ This lack of complete reactivity might preclude passivation of the entire surface and therefore result in decreased stability after substantially longer term exposure to air.

The work described above provides evidence of a chemically functionalized Si surface that has a stable, low surface recombination velocity in air. The intensity of the light pulse at high-level injection was sufficient to eliminate essentially any equilibrium potential drop that might exist in the solid. We therefore conclude that the changes in the observed carrier recombination lifetime are primarily due to changes in surface state density and/or surface-trap carrier-capture rate constants, as opposed to changes in the electrostatic surface potential. The latter effect has been observed for native $\mathrm{Si}$ surfaces in contact with $\mathrm{NH}_{3}$ and oxidizing ambients. ${ }^{23,24}$ The alkylation methodology could prove useful in providing passivation layers for Si nanoparticles, for Si surfaces in photovoltaic devices, and for other novel applications of Si surfaces in electrical device structures where low recombination velocity surfaces are beneficial.

In summary, we have demonstrated that it is possible, through molecular level control over surface chemistry, to produce Si surfaces that are highly electrically passive and which are far more stable in ambient air than $\mathrm{H}$-terminated $\mathrm{Si}$ surfaces. Assuming a geometric cross section for carrier capture by surface traps of $1 \times 10^{-15} \mathrm{~cm}^{2}$, the observed surface recombination velocity of $2 \times 10^{1} \mathrm{~cm} \mathrm{~s}^{-1}$ for methylterminated $\mathrm{Si}$ surfaces translates into only one active electrical defect for every $\approx 250000$ surface atoms. The electrical properties of these alkylated surfaces in air correlate well with the improved electrochemical properties of these surfaces in contact with nonaqueous and aqueous electrolytes, and with the improved resistance to oxidation observed pre- viously for such alkylated Si surfaces. ${ }^{14,15}$ The long-term stability of the various alkylated surfaces in air is currently under investigation, as are other routes to surface functionalization that will be equally electrically passive yet allow further elaboration of the chemical and physical properties of overlayers on the Si surface.

The authors acknowledge the National Science Foundation, Grant No. CHE-9974562, for support of this work.

${ }^{1}$ E. Yablonovitch and T. J. Gmitter, Solid-State Electron. 35, 261 (1992).

${ }^{2}$ W. D. Eades and R. M. Swanson, J. Appl. Phys. 58, 4267 (1985).

${ }^{3}$ S. M. Sze, Physics of Semiconductor Devices, 2nd ed. (Wiley, New York, 1981).

${ }^{4}$ S. J. Fonash, Solar Cell Device Physics (Academic, New York, 1981).

${ }^{5}$ E. Yablonovitch, D. L. Allara, C. C. Chang, T. Gmitter, and T. B. Bright, Phys. Rev. Lett. 57, 249 (1986).

${ }^{6}$ A. Bansal, X. Li, I. Lauermann, N. S. Lewis, S. I. Yi, and W. H. Weinberg, J. Am. Chem. Soc. 118, 7225 (1996).

${ }^{7}$ M. R. Linford, P. Fenter, P. M. Eisenerger, and C. E. D. Chidsey, J. Am. Chem. Soc. 117, 3145 (1995)

${ }^{8}$ M. R. Linford and C. E. D. Chidsey, J. Am. Chem. Soc. 115, 12631 (1993).

${ }^{9}$ A. V. Teplyakov, M. J. Kong, and S. F. Bent, J. Am. Chem. Soc. 119, 11100 (1997).

${ }^{10}$ C. H. de Villeneuve, J. Pinson, M. C. Bernard, and P. Allongue, J. Phys. Chem. B 101, 2415 (1997).

${ }^{11}$ J. M. Buriak and M. J. Allen, J. Am. Chem. Soc. 120, 1339 (1998).

${ }^{12}$ J. M. Buriak, M. P. Stewart, T. W. Geders, M. J. Allen, H. C. Choi, J. Smith, D. Raftery, and L. T. Canham, J. Am. Chem. Soc. 121, 11491 (1999).

${ }^{13}$ C. Gurtner, A. W. Wun, and M. J. Sailor, Angew. Chem. Int. Ed. Engl. 38, 1966 (1999).

${ }^{14}$ A. Bansal and N. S. Lewis, J. Phys. Chem. B 102, 4058 (1998).

${ }^{15}$ A. Bansal and N. S. Lewis, J. Phys. Chem. B 102, 1067 (1998).

${ }^{16}$ G. S. Higashi, R. S. Becker, Y. J. Chabal, and A. J. Becker, Appl. Phys. Lett. 58, 1656 (1991).

${ }^{17}$ T. Tiedje, J. I. Haberman, R. W. Francis, and A. K. Ghosh, J. Appl. Phys. 54, 2499 (1983).

${ }^{18}$ E. Yablonovitch, R. M. Swanson, W. D. Eades, and B. R. Weinberger, Appl. Phys. Lett. 48, 245 (1986).

${ }^{19}$ E. Yablonovitch, C. J. Sandroff, R. Bhat, and T. Gmitter, Appl. Phys. Lett. 51, 439 (1987).

${ }^{20}$ M. D. E. Forbes and N. S. Lewis, J. Am. Chem. Soc. 112, 3682 (1990).

${ }^{21}$ M. Kunst and A. Sanders, Semicond. Sci. Technol. 7, 51 (1992).

${ }^{22}$ A. Fidélis, F. Ozanam, and J. N. Chazalviel, Surf. Sci. 444, L7 (2000).

${ }^{23}$ T. M. Buck and F. S. McKim, J. Electrochem. Soc. 343, 709 (1963).

${ }^{24}$ A. Many, Y. Goldstein, and N. B. Grover, Semiconductor Surfaces (North Holland, Amsterdam, 1965). 